\title{
Hugo Chávez: de la idea de confederación de estados a la conformación de la alianza bolivariana para los pueblos de nuestra america*2 $^{* 2}$
}

\section{Hugo Chávez: da ideia da confederação de Estados à conformação da Aliança Bolivariana dos povos da América}

Eudis F. Fermín T. ${ }^{1}$

\section{Resumen}

En el estudio se analiza los elementos políticos y diplomáticos de la Alianza Bolivariana para los Pueblos de Nuestra América (ALBA), una estrategia unionista contrahegemónica, presentada primero discursivamente, idea de creación de la Confederación de Estados, por el presidente Chávez en los foros latinoamericanos y caribeños durante la etapa 1999-2003, que iniciaría su construcción organizacional e institucional entre el 2004 y 2010 como proyecto político continental, período éste en que logró un posicionamiento geoestratégico para promover e impulsar el desarrollo socio-productivo y las relaciones comerciales de los pueblos. Es un estudio analítico-documental. El presidente Chávez pretende con esta estrategia desmontar la razón del mercado que predomina en la concepción integracionista de los gobiernos latinoamericanos y en los espacios públicos de integración, revelando sus contribuciones nefastas para el aumento de los índices de pobreza y exclusión social en la región, en contradicción al desarrollo social prometido por la derecha promotora del neoliberalismo. Se concluye que en la nueva etapa del proceso de transformación y cambio continúo que está experimentando el continente en las primeras décadas del siglo XXI, el gobierno de Chávez esta operacionalizando este enfoque a través de alianzas estratégicas con gobiernos que comparten en su diplomacia presidencial elementos ideológicos y políticos similares para la instauración del nuevo socialismo del siglo XXI.

* Recebido em: $16 / 02 / 2012$ Aprovado em 25/04/2012

1 Licenciado en Ciencias Políticas y Administrativas, egresado de la Universidad Rafael Urdaneta (URU). Sociólogo, egresado de la Universidad del Zulia (LUZ). Investigador Libre en el Área de Análisis Político y Relaciones Internacionales. Consultor en organizaciones públicas. E-mail: eudisfermin@gmail.com. Es Profesor de la Universidad Nacional Experimental de las Fuerzas Armadas, UNEFA-Núcleo Portuguesa (Venezuela).

2 El papel de trabajo se inscribe en el Sub-eje La Emergencia de Nuevos Procesos de Integración en la Región, correspondiente al Eje Temático Tratados de Libre Comercio y Planes Geoestratégicos, del XII Congreso Internacional sobre Integración, Regional, Fronteras y Globalización en el continente Americano, a celebrarse en Quito (Ecuador), los días 23, 24 y 25 de Noviembre de 2011
Palabras-Claves: Hugo Chávez. Estrategia Unionista. Mercado. Pueblos.

\section{Resumo}

Este estudo analisa os elementos políticos e diplomáticos da Aliança Bolivariana para os Povos da América (ALBA), uma estratégia de integração contra-hegemônica, apresentada pela primeira vez pelo Presidente Hugo Chávez, por meio da ideia de criação da Confederação dos Estados, nos fóruns latino-americanos e caribenhos durante o período de 1999-2003. A ALBA iniciaria sua construção organizacional e institucional entre o período de 2004 e 2010, como projeto político continental, período em que conseguiu um posicionamento geoestratégico para promover e impulsionar o desenvolvimento sócio-produtivo e as Relações comerciais dos povos. Trata-se de um estudo analítico-documental. O Presidente Chávez pretende 
com esta estratégia desmontar a razão do mercado que predomina na concepção integracionista dos governos latino-americanos e nos espaços públicos de integração, revelando suas contribuições nefastas para o aumento dos índices de pobreza e exclusão social da região, em contradição com o desenvolvimento social prometido pela direita promotora do neoliberalismo. Conclui-se que, na nova etapa do proceso de transformação e mudança contínua que o continente está experimentando nas primeras décadas do século XXI, o governo de Chávez está operacionalizando este enfoque através de alianças estratégicas com governos que compartilham, em sua diplomacia presidencial, elementos ideológicos y políticos similares para a instauração do novo socialismo do século XXI.

Palavras-chave: Hugo Chávez. Estratégia de Integração. Mercado. Povos.

\section{Introducción}

Desde 1990 en los mecanismos e instancias de los espacios públicos de integración, el tema central de la agenda era económico, principalmente en las relaciones comerciales y financieras, mientras que la pobreza y la exclusión social estaban delegadas a un segundo plano, la reestructuración neoliberal imposibilitaba que regresara a corto plazo a la agenda como tema prioritario; el índice pobreza y exclusión social incrementó progresivamente, en la medida en que los sectores más vulnerables de la población caían en el abismo de la miseria en un contexto de recuperación de las variables macro-económicas. Así, con el Consenso de Washington los sectores transnacionales, en plena alianza con la clase política norteamericana, aumentaron su capital, para fortalecerlo impulsaron en el hemisferio desde 1994 la propuesta de creación del Área de Libre Comercio de las Américas (ALCA). Es a finales de la década que algunos gobiernos se distanciarían moderadamente o rechazarían la propuesta, presentando nuevas opciones con proyectos de integración: la Alternativa Bolivariana para las Américas (ALBA) del gobierno revolucionario de Venezuela (transformada ideológicamente el 24 de junio de 2009 en Aliaza, conservando la misma sigla), el Plan Puebla-Panamá (PPP), la alianza entre México y los países centroamericanos, al cual se incorporaría Colombia, ${ }^{3}$ y la Comunidad Sudamericana de Naciones (hoy día Unión de Naciones Sudamericanas, UNASUR), 12 gobiernos sudamericanos se comprometieron en avanzar hacia la convergencia de los espacios públicos de integración económica imperantes. ${ }^{4}$

En el caso específico del primer proyecto de integración, surgiría en el continente al ritmo del nacimiento de los gobiernos democráticos anti-sistemas, los calificados por algunos especialistas, unos de nueva izquierda (PARAMIO, 2006) y otros, centroizquierda (PETRAS, 2008). El presidente venezolano Hugo Chávez, es el autor de éste proyecto, preocupado por la situación socio-económica de los pueblos latinoamericanos y caribeños, originada por las consecuencias de la implementación del código económico de Washington y las relaciones internacionales inter-estatales unipolar, promueve en su política exterior 1999-2001 una opción unionista diametralmente opuesta a la propuesta hemisférica norteamericana, la creación de la Confederación de Estados, que sería bautizada en la III Cumbre de Estados del Caribe, celebrada en diciembre de 2001, con el nombre de Alternativa Bolivariana para las Américas, ALBA: un proyecto revolucionario de alcance continental con una fuerte carga política e ideológica de rechazo al ALCA y a los

\footnotetext{
3 El distanciamiento moderado de los Estados que suscribieron el Plan Puebla-Panamá: México, Nicaragua, Honduras, Guatemala, Panamá, Costa Rica, El Salvador y Belice, no era más que una estrategia mexicana para incorporarlos silenciosamente en el libre mercado pregonado por el ALCA. En la X Cumbre del Mecanismo de Dialogo y Concertación de Tuxtia, celebrada el día 28 de junio de 2008, acordaron en la Declaración de Villahermosa que el programa de mesoamericano de integración y desarrollo Plan Puebla-Panamá, modifica su nombre original para denominarse Proyecto de Integración y Desarrollo de Mesoamerica o Proyecto Mesoamerica. A la cumbre asistieron los gobiernos de México y los centroamericanos, Colombia se adhiere como miembro pleno. El PPP es una estrategia que busca el aprovechamiento de las ventajas comparativas naturales del sur-sureste de México y de la región centroamericana, que se inscribe en los preceptos del neoliberalismo, bajo la hegemonía norteamericana, al centrarse en el control de áreas geográficas claves para su explotación, los recursos naturales y en la mano barata (Sandoval, 2003), contribuyendo, en el sentido publicitario, al desarrollo humano.

4 En cada uno de estos nuevos esquemas de integración económica surgen liderazgos políticos propios, uno, Brasil (UNASUR) y el otro, México (PPP). Desde la óptica del presidente Chávez estos esquemas responden uno más que el otro a la lógica del capitalismo, pero se inclina por la UNASUR, pretendiendo moldearlo con el enfoque del ALBA y sus relaciones geo-estratégicas con Brasil y Argentina dentro de la UNASUR, y con los gobiernos miembros de éste espacio y del ALBA, Bolivia y Ecuador.
} 
tratados de libre comercio; según el presidente Chávez estas estrategias neoliberales son reproductoras de la desigualdad social en los pueblos de las américas. Visto así, el ALBA y el ALCA son dos formas contrapuestas de ver el mundo, una revolucionaria, lucha contra la pobreza y la exclusión social, y la otra, neoconservadora, incentivadora del consumismo y de la razón del mercado por encima de la racionalidad política del Estado, con el objetivo de instaurar y consolidar el libre mercado.

Desde esta perspectiva, en el estudio se analiza los elementos políticos y diplomáticos del ALBA, una estrategia unionista contrahegemónica, presentada discursivamente por el presidente Chávez en los foros latinoamericanos y caribeños celebrados durante la etapa 1999-2003, iniciaría su construcción institucional entre el 2004 y 2010 como proyecto político continental; en estos períodos los elementos -políticos y diplomáticos-, bajo el enfoque de la revolución bolivariana, lograron activar un proceso de posicionamiento geo-estratégico para promover e impulsar el desarrollo humano de los pueblos. La estrategia bolivariana pretende modificar sustancialmente la concepción sobre la integración económica instaurada desde la década de 1950 y renovada por los postulados del neoliberalismo, ya que generó continuidad en los espacios públicos de integración, con las directrices del Consenso de Washington, al desarrollo económico en las políticas gubernamentales concertadas en el multilateralismo regional; cuando el Sistema de Integración Centroamericano (SICA), la Comunidad del Caribe (CARICOM), la Comunidad Andina de Naciones (CAN) y el Mercado Común del Sur (MERCOSUR), iniciaron un proceso de transformación gradual hacia una economía competitiva, que incluye la reformulación de su filosofía de gestión y los objetivos estratégicos para llegar más allá de las normas aduaneras y comerciales, incorpoar el libre mercado en las relaciones económicas entre los países.

El ALBA, como proyecto político continental, marca distancia con el libre mercado, cuando su promotor fundacional, el presidente venezolano Hugo Chávez, revela críticamente, en los foros latinoamericanos y caribeños en los que asiste, los resultados de las medidas económicas neoliberales en los pueblos, el aumento de la pobreza y exclusión social, paradójicamente como contrapeso a la estabilidad de las variables macroeconómicas alcanzadas en la década de 1990, entre ellas el producto interno bruto, las reservas internacionales, la reducci- ón del déficit del gasto público y el control de la inflación. ${ }^{5}$ Los primeros intentos de llevar el enfoque de su propuesta estarían dirigido al MERCOSUR, estrechando relaciones políticas, económicas y energéticas con las dos principales economías de Sudamérica, Brasil y Argentina, y con los países de la CAN, revisando los acuerdos económicos celebrados y buscando instaurar en éste esquema el bolivarianismo con acciones concretas, por ejemplo, la propuesta de un pasaporte único y de una sola moneda, presentadas en la Cumbre Presidencial Andina de 2001. Estos intentos se lograron en cierta medida en el primer caso, a pesar de que ningunos de los países del MERCOSUR pertenecen al ALBA, pero fracasaron con la CAN, al punto de que Venezuela se retiró en abril de 2006, en vista del Tratado de Libre Comercio (TLC) que negociaban los gobiernos de Colombia y Perú con los Estados Unidos, pero siguió con las relaciones bilaterales con Bolivia (2006) y Ecuador (2007) para el fortalecimiento político y comercial del espacio público unionista.

\section{El presidente Chávez: ideas, propuesta y estrategia unionista}

Para algunos académicos y analistas de las relaciones internacionales, la orientación de la propuesta a la estrategia unionista venezolana consiste en la reivindicación del pensamiento bolivariano entorno a la idea de la Patria Grande de América. Idea que buscó a materializarse una vez culminada la Batalla de Ayacucho, cuando el Libertador Simón Bolívar se dedicó a la construcción de la viabilidad del proyecto de confederación hispanoamericana, teniendo siempre presente la necesidad de lograr la independencia de Costa Rica y Cuba, para frenar las pretensiones imperiales europeas y las de Estados Unidos hacia las nacientes naciones (MARTÍNEZ, 1957). La primera huella histórica se registra aquel 7 de abril de 1824, fecha en que el Libertador envía a los gobiernos hispanoamericanos independizados, una carta de invitación para que asistieran a un congreso plenipotenciario. Los asistentes al Congreso Anfictiónico de Panamá de 1826

\footnotetext{
5 En el Anuario Estadístico de América Latina y el Caribe correspondiente al año 2000, elaborado por el Equipo Técnico de la Comisión Económica para América Latina y el Caribe (2000), CEPAL, se observa el comportamiento de estas variables macroeconómicas de los países latinoamericanos y caribeños durante el período 1990-1999.
} 
terminaron discutiendo y aprobando concepciones diferentes a las instruidas por el Libertador Simón Bolívar (AGUIRRE, 2007). El trabajo del Congreso Anfictiónico de Panamá quedó inconcluso, con la estrategia unionista desplegada por el presidente Chávez durante el período 2004-2010 se pretendía retomarlo y convertirlo en una realidad política y social, que condicionara las relaciones mercantilistas a los intereses de los pueblos, erradicar la pobreza y la exclusión social.

Los fundamentos históricos y filosóficos del proyecto político continental del presidente Chávez se sostiene en los ideales de unificación política del Libertador Simón Bolívar (NAÍM; FERMÍN, 2002), ideales éstos que compartieron y coincidieron varios próceres y héroes de la gesta independentista hispanoamericana, quienes configuraron conjuntamente un pensamiento unionista en lo político más que en lo económico. Este pensamiento se mantuvo relativamente vigente en la última Confederación de Estados del siglo XIX, los políticos conservadores y liberales lo convertirían en un discurso retórico que fue abriendo paso a los modelos de integración económica instaurados en el continente a partir de la segunda mitad del siglo XX. En otras palabras, los orígenes históricos y filosóficos del ALBA se encuentran en el pensamiento bolivariano de unificación política, como también en los ideales de los otros precursores, como Juan Martín de Pueyrredón, Bernardo O'Higgins, José de San Martín, Bernardo Monteagudo (MARTíNEZ, 1957), y, en un hombre que divulgó en sus escritos los postulados de la lucha por la independencia a finales del siglo XIX, el Apóstol José Martí, el autor de la frase celebre Nuestra América. ${ }^{6}$ La alternativa bolivariana recoge y expresa un sentimiento histórico olvidado en las políticas exteriores de los gobiernos de derecha y de la vieja izquierda, un compromiso con el pasado desde el presente para reconstruir en el futuro inmediato la Gran Patria Americana.

Según el presidente Chávez la estrategia unionista desplegada con el ALBA y sus organismos especializados ${ }^{7}$ tiene una orientación diametralmente opuesta a las estra-

6 Sobre este último aspecto -Nuestra América-, véase Cintio Vitier (2006). Vida y obra del Apóstol José Martí. La Habana (Cuba): Fondo Cultural del ALBA.

7 Se consideran organismos especializados del espacio público unionista a Petro-Caribe, Tele-Sur, Banco del ALBA y la Universidad del ALBA (UNIALBA), en proceso de creación, con la coordinación de los ministerios de educación de los países miembros. tegias integracionistas iniciadas desde mediado del siglo XX en América Latina y el Caribe; producto de las contradicciones del capitalismo y la clase gobernante dominante, presenta esta estrategia, para que el Estado garantice la igualdad de condiciones y oportunidades a los ciudadanos de la Gran Patria Americana. De manera que la estrategia bolivariana se orienta hacia el fortalecimiento social del Estado, atendiendo las consecuencias sociales de la transnacionalización del comercio y la globalización de la economía latinoamericanas y caribeñas, no en el sentido del libre mercado, sino con un enfoque económico justo y equitativo: la complementariedad económica como lo anuncian los presidentes en las declaraciones bolivarianas. Por ello, en las reuniones con los burócratas, políticos y empresarios exige la implementación de este enfoque; sus postulados descifran las distancias históricas de las directrices del modelo desarrollista, promovidas por la CEPAL, y la sustitución de éstas por las del Consenso de Washington. Busca que los Estados latinoamericanos y caribeños, así como se comprometieron con los organismos multilaterales internacionales en responder a la crisis del déficit fiscal con los créditos otorgados por éstos, se comprometan con el desarrollo humano de los pueblos a través de los proyectos gran-nacionales y la creación de instituciones económicas regidas por la pertinencia social, no la lógica de la rentabilidad en los negocios.

\section{De una política exterior de integración neoliberal a una unionista con enfoque humanista, endógeno y socialista}

Desde que el gobierno de Carlos Andrés Pérez anuncia las medidas económicas en febrero de 1989, el formato de política exterior que predominó en Venezuela durante el período 1959-1988, experimenta un proceso sustantivo de modificación de su visión de integración económica, esto es, en las directrices del desarrollismo cepalino que se configuraron en la estructura estatal de las relaciones internacionales, sobre la base de la premisa de que el desarrollo económico armónico y diversificado de los países latinoamericanos y caribeños agrupados en los espacios públicos de integración. Esta esencia desaparece agresivamente de estas instancias decisionales de la política exterior y de la diplomacia presidencial, cuando comienza la reducción del tamaño del Estado, la intervención estatal en la economía, la eliminación de los subsidios al sector empresarial y la privatización de las em- 
presas públicas, todas estas medidas fueron tomadas con la adopción de los fundamentos del neoliberalismo, que tenía una doble direccionalidad, una, la del carácter nacional de estas medidas y la otra, las implicaciones de las mismas en los procesos de integración, para implementar la razón del mercado en el imaginario socio-político y técnico de los operadores de las relaciones comerciales y de la política exterior.

A partir de diciembre de 1994 hasta febrero de 1999, Venezuela asumió una posición de "reconcomio diplomático" a la propuesta hemisférica de integración neoliberal, presentada por los Estados Unidos en la I Cumbre de las Américas: el ALCA, ${ }^{8}$ en el fondo era contra el gobierno de Bill Clinton por la jugada hecha en la elección del Secretario de la Organización de los Estados Américanos (OEA) (ROMERO, 2006). Pero cuando Chávez llega al poder estatal, el 6 de diciembre de 1998, primero, crítica fuertemente al ALCA, proponiendo una revisión exhaustiva de sus fundamentos económicos y efectos en los escenarios sub-regionales de economías asimétricas, propone adoptar un "capitalismo con rostro humano", la llamada "Tercera Vía"; y, luego, comienza acciones gubernamentales para el desplazamiento de la propuesta norteamericana del escenario hemisférico, evitar su implementación, cambio que promueve el posicionamiento estratégico del proyecto político continental de la revolución bolivariana, el cual se funda sobre la premisa rectora del desarrollo humano integral de los pueblos. En otras palabras, la estrategia unionista bolivariana genera una ruptura paradigmática de los formatos de los espacios públicos de integración imperante-MERCOSUR, CAN, SICA, CARICOM-, promotores del desarrollo económico, quedando subordinado a éste el desarrollo humano, sin descuidar la economía, pero ahora vista bajo la lógica de la razón del Estado (FERMíN, 2009).

\footnotetext{
8 Para los Estados Unidos la nueva realidad económica mundial de la década de 1990, demandaba en su "patio trasero", la creación de un esquema hemisférico de integración económica, capaz de competir con la Comunidad Europea y Asia. La avanzada de la Comunidad Europea y los “Tigres Asiáticos” en los mercados económicos centroamericanos, y, en algunos países sudamericanos y caribeños durante ésta época, tenía que preocupar a las empresas trasnacionales norteamericanas que invertían en la región en diferentes sectores de la economía, el acercamiento comercial y de cooperación para el desarrollo de esos países, aún más por la situación futura de las condiciones de las relaciones comerciales con los países latinoamericanos en el contexto de la globalización de la economía.
}

La propuesta ALCA establecía a Venezuela desafíos económicos superables. Ante de la llegada de Chávez al poder estatal, en la política exterior del gobierno de Rafael Caldera (1994-1999), más allá del "reconcomio diplomático", no se negaba que existiera motivos suficientes para que se aceptara la referida propuesta de libre comercio, previa negociaciones, un acuerdo comercial con Estados Unidos; según la opinión de varios expertos, fortalecería las relaciones comerciales del país, específicamente las importaciones de bienes y servicios provenientes del país destino y las exportaciones, ya que es un socio natural de Estados Unidos; en el caso de la exportación de petróleo, este ingresa al mercado estadounidense casi libre de aranceles, por considerarlo un tema de prioridad para ambos países, fue excluido de la agenda de negociación del ALCA. Esta situación pasa de un extremo a otro con el gobierno revolucionario, cuando el presidente Chávez asiste a las cumbres de las Américas, manifiesta públicamente las posibilidades de la exclusión unilateral del acuerdo

la opción de excluirse no es algo absolutamente irracional, pues el ALCA, en la forma que lo plantea Estados Unidos, es un ejemplo del modelo de integración profunda de tipo norte-sur, es decir, un acuerdo entre países desarrollados y en desarrollo, en los cuales, además de la degradación arancelaria, se negocian temas 'relacionados con el comercio', que son bastantes sensibles para los países en desarrollo (RUIZ, 2007, p. 29).

Es evidente que desde 1999 con el gobierno del presidente Chávez se da un cambio cualitativo en la política exterior venezolana de integración; dicho en palabras de James Petras (ESCORCIA et al, 2007, p. 83)

Es en su política exterior que podríamos decir
que Chávez, de manera consistente ha adopta-
do posturas radicales. Radicales en el sentido
de que rechaza las agresivas políticas de Estados
Unidos, critica y se opone al [...] ALCA [...], y
esta buscando formar algún tipo de alianza con
otros gobiernos disidentes en Latinoamericana.

Este cambio, opera con mayor fuerza al ritmo de la dinámica de cinco acontecimientos socio-políticos, desafíos, que determinaron la re-orientación ideológica de la política exterior de la revolución bolivariana: i) los trágicos sucesos de abril de 2002, cuando la huelga nacional convocada por el sector opositor capital-trabajo termina con un golpe de Estado y en menos de 48 horas retorna Chávez al poder estatal; ii) el paro petrolero, conocido también como el sabotaje petrolero, iniciado en diciem- 
bre de 2002 y suspendido por el gobierno en febrero de 2003; iii) la oportunidad de la oposición para salir democráticamente de Chávez, el referendo revocatorio del mandato presidencial, el 15 de agosto de 2004, en el cual triunfa Chávez; iv) las elecciones presdienciales del 3 de diciembre de 2006 y el inicio de la construcción del Nuevo Socialismo del Siglo XXI; y, v) la reforma del artículo 230 de la CRBV, la aprobación de la reelección presidencial en la enmienda constitucional del 15 de febrero de 2009. Este últmo acontecimiento sirvió para profundizar la re-orientación de la política exterior a la postura anti-imperialista y anti-capitalista, como se observa en los discursos de Chávez y en las declaraciones de las cumbres del ALBA (2009b; 2009c; 2009d; 2009e).

En los foros internacionales Chávez denuncia la inherencia de Estados Unidos en los asuntos internos de Venezuela, pasando por el golpe de Estado hasta llegar al referendo revocatorio del mandato presidencial, para evitar que se desarrolle el proyecto político continental de la revolución bolivariana, con el propósito de que un gobierno de derecha garantizara el control del petróleo venezolano a las empresas transnacionales norteamericanas. Es por esto que en la III Cumbre de las América, se niega a firmar las clausuras referentes al concepto de democracia representativa y la necesidad de implementar una economía de libre mercado hemisférica; llama a los gobernantes que participan en el evento a la reflexión, concluye en que había que decir no al ALCA, ya que su implementación implicaría el aumento del índice de la pobreza crítica, la tasa de desempleo e inflación, favoreciendo sólo a las empresas transnacionales y la economía norteamericana, a la "oligarquía transnacional", legitimadora de la lógica del capital global y de la pobreza de los pueblos, los excluidos de los excedentes de las negociaciones y las ganancias de la comercialización del petróleo. En pocas palabras, después del retorno de Chávez al poder estatal en abril de 2002, según Pérez (2009, p. 154),

se dedicó a impulsar una política exterior de oposición a EE.UU., orientada a propiciar el cambio de mapa político regional, a utilizar el petroleo en programas de cooperación y como arma política, así como introducir actores extraregionales.

Para que la propuesta del ALBA no quedara en un discurso humanista retórico, comienza a preparar el terreno para implementar la estrategia unionista en un escenario latinoamericano caracterizado por una práctica política conservadorada. El 14 de diciembre de 2004, una vez superado los tres primeros grandes desafíos políticos mencionados, el presidente Chávez, con su único aliado político e ideológico revolucionario, Fidel Castro, firman en la ciudad de La Habana el Acuerdo de Aplicación del ALBA. Se didicó particular atención a la estructuración organizativa de la alternativa bolivariana, y a las acciones políticas y diplomáticas (comerciales y petroleras), para promover las potencialidades económicas de Venezuela en las "relaciones interamericanas bolivarianas", por esto, el presidente Chávez consideró necesario la creación del Ministerio de Comercio Exterior e Integración en el año 2005, para que se encargara, en coordinación directa con el Ministerio de Relaciones Exteriores y PDVSA, el posicionamiento del ALBA en las áreas geo-estratégicas del continente, con miras a la construcción del sistema internacional multipolar, como se estableció en Los Lineas Generales del Plan de Desarrollo Social y Económico de la Nación 2001-2007 (MINISTERIO DE DESARROLLO Y PLANIFICACIÓN, 2001).

Seriá después del 3 de diciembre de 2006, que se iniciaría la conformación institucional y organizacional de la estructura funcional del ALBA, además, se incorporía otros países que comparten una misma ideología política con los postulados de la revolción bolivariana, como Bolivia (2006), Nicaragua (2007), Honduras (2008), Ecuador (2009) y varios países caribeños, ellos son: Comunidad de Dominica (2008), San Vicente y las Granadinas (2009) y Antigua y Barbuda (2009). En otras palabras, es a partir del año 2006 que algunos gobiernos electos apoyan el proyecto político revolucionario, incorporándose al ALBA, y otros que han celebrando acuerdos bilaterales con Venezuela, fuera de los tradicionales espacios públicos de integración, no han manifestado interés en convertirse en miembros plenos de la alternativa bolivariana, como se observa en la actitud de los gobiernos de Brasil, Argentina, Uruguay, Paraguay y Ecuador hasta junio de 2009, aliados estratégicos de la revolución bolivariana en el continente.

\section{ALBA: motor de la emancipación de los pueblos}

Desde el origen de la revolución bolivariana esta presente la idea de una nueva estrategia de integración, en este planteamiento el centro de preocupación y ocupación 
de Chávez esta en la lucha contra la pobreza y exclusión social de los pueblos, asociándolo así con los ideales del Libertador Simón Bolívar referente a la "suprema felicidad social”. Esto explica que el candidato Chávez en su programa de gobierno, titulado La Propuesta de Hugo Chávez para Transformar a Venezuela (MOVIMIENTO QUINTA REPÚBLICA, 1998), presentado al electorado en noviembre de 1998, delinea las primeras ideas para la formulación e implementación de esta estrategia, que serían incorporadas luego con la categoría comunidad de naciones en el artículo 153 de la Constitución de la República Boliviana de Venezuela de 1999, otorgando jurídicamente un carácter supranacional; la estrategia sigue presente en el programa de gobierno de 2000, La Propuesta de Hugo Chávez para continuar la Revolución (MOVIMIENTO QUINTA REPÚBLICA, 2000), con el cual triunfa en las elecciones presidenciales del 30 de julio de éste año. En este último programa, se estructura las Líneas Generales del Plan de Desarrollo Económico y Social de la Nación 2001-2007 (MINISTERIO DE DESARROLLO Y PLANIFICACIÓN, 2001), de su segundo período de gobierno, reformulado con los objetivos estratégicos de la nueva etapa de la revolución, definidos en noviembre de 2004; ${ }^{9}$ y, en la propuesta electoral que presentó en las elecciones presidenciales del 3 de diciembre de 2006, las Siete Líneas Estratégicas del Proyecto Nacional Simón Bolívar 2007-2013, el denominado Primer Plan Socialista de la Nación, como lo califica el mismo gobierno, aparece en el Enfoque Nueva Geopolítica Internacional (MINISTERIO DE DESARROLLO Y PLANIFICACIÓN, 2007).

Con el ALBA el gobierno de Chávez pretende desmontar en los Estados miembros la razón del mercado, que

9 Después del triunfo de Chávez en el referendo revocatoria del mandato presidencial, el 15 de agosto de 2004, redimensiona política e ideológicamente los fundamentos de la revolución bolivariana en la reunión de alto nivel celebrada los días 12 y 13 de noviembre de éste mismo año, cuando definen los 10 objetivos estratégicos, el nuevo mapa estratégico de la revolución, la nueva etapa de la revolución bolivariana (EL TROUDI, 2005). En el objetivo estratégico número 10 sostiene "seguir impulsando el nuevo sistema multipolar internacional”, entre los objetivos específicos, está "continuar impulsando el modelo de integración alternativa (ALBA)" y las herramientas para lograrlo son la creación de nuevas organizaciones promotoras de la integración alternativa, en áreas como la energética (Petro-Caribe), comunicacional (Tele-Sur) y educativa (Universidad del Sur), luego se le agregaría la entidad bancaria (Banco del ALBA), y, en el ámbito organizacional de la alianza la Universidad del ALBA (UNIALBA). predomina en la concepción integracionista de los gobiernos latinoamericanos, explica en los foros internacionales sus efectos nefastas para los sectores más vulnerables de las sociedades latinoamericanas y caribeñas: el individuo queda desaparado, se debe grantizar el mismo su bienestar social sin la intervención estatal; por esto afirma Chávez en las cumbres bolivarianas que fue un error la decisión de los gobiernos, los de la década de 1990 y de aquellos que siguen conservando el modelo neoliberal, en implementar en los sistemas económicos latinoamericanos las políticas de reducción del Estado y de inclusión de las áreas tradicionales del sector público en el mercado, para recuperar la estabilidad de las variables macroeconómicas (reservas internacionales, PIB, inflación). Esto explica porque exhortó durante el período 1999-2003 a los gobiernos de turnos a revisar y analizar los enfoques de integración imperantes en la región y a reconocer en esos esquemas a los pueblos con una economía justa y equitativa. Por esto, Chávez trazaría durante el período 2004-2010 una estrategia unionista con una visión humanista, endógena y socialista de las relaciones comerciales y económicas entre los pueblos, celebrando y ejecutando acuerdos de cooperación comercial, tecnológica y energética con los países sudamericanos, centroamericanos y caribeños, incluso con aquellos que ha tenido fuertes tensiones y conflictos diplomáticos, como Colombia, Perú, México y Costa Rica.

En el período 1999-2003, el proyecto ALBA no avanza ni en lo político ni en lo diplomático, queda en una propuesta sólo expuesta a los gobernantes, políticos, empresarios, estudiantes y movimientos sociales latinoamericanos y caribeños, si se compara con la evolución de la propuesta del presidente mexicano Vicente Fox, el PPP, materializada en agosto de 2000, en el acuerdo firmado con los países centroamericanos; ${ }^{10}$ no despertó interés en los gobernantes, ni en los llamados progresistas, Argenti-

\footnotetext{
10 "Como es sabido, este acuerdo deriva de un compromiso asumido por el presidente mexicano Vicente Fox durante su campaña electoral, y tiene como propósito logar inversiones importantes en las regiones menos desarrolladas del sur de su país (...) El acuerdo abarcó también a Panamá para logar un ámbito geográfico extenso y de mayor influencia de México en esta subregión. Originalmente se acordó un conjunto de iniciativas entre los siete países, que abarcó aspectos de infraestructura, electrificación, turismo y asuntos sociales" (De la Ossa, 2003: 64). La PPP implica un financiamiento de los diversos proyectos en los aspectos indicados, que contaron con el financiamiento del gobierno mexicano y el respaldo en los organismos financieros internacionales.
} 
na y Brasil, con quien Venezuela estrechó más relaciones económicas que políticas en éste período. El presidente Chávez analiza con su equipo de política exterior los obstáculos y dificultades para la conformación multilateral de la propuesta, decide por la opción bilateral, por lo que inicia desde mediados del año 2004, en el contexto de pleno conflicto político doméstico, con los gobiernos y movimientos sociales de la misma línea política, las acciones respectivas para la creación del ALBA; el primero en establecer las negociaciones para impulsar la estrategia unionista fue con la Cuba de Fidel Castro, en septiembre de 2004, y en diciembre de éste mismo año, teniendo ambos gobiernos una clara visión de sus potencialidades políticas y energéticas en la región, firmaron un ambicioso acuerdo de cooperación integral, en el cual abarcaba diferentes sectores económicos y sociales, instaurando una especie de "diplomacia solidaria entre los pueblos".

Chávez y Fidel, con la estrategia unionista bolivariana, lograron comprender que lo primero es atender la naturaleza humana de los individuos, las necesidades sociales en el sentido de configurar en el imaginario socio-político latinoamericano y caribeño la construcción de la igualdad de condiciones y oportunidades socio-económicas para el colectivo, por encima del "progreso o desarrollo económico", sin obviar o ignorar la importancia del mercado en el mundo de la globalización y transnacionalización; conviertieron, la máxima bolivariana, la suprema felicidad social, en una expresión de la realidad y sentimiento popular, con atención en salud, educación, vivienda y alimento, resaltando en el comportamiento colectivo que los gobiernos revolucionarios promueven la igualdad de condiciones y oportunidades para los ciudadanos, sobre todo en los estratos sociales más vulnerables de la población (Equipo de Investigación del Instituto de Altos Estudios Diplomáticos Pedro Gual, 2006). Bajo esta orientación, al ALBA se fueron adhiriendo los nuevos gobiernos de izquierda, los que ganaron las elecciones presidenciales en Bolivia (2006), Nicaragua (2007), Ecuador (2009) y otros que no eran de izquierda, el de la Comunidad de Dominica (2008), Honduras (2008), San Vicente (2009) y Barbado (2009).

Desde este enfoque, se promueve y se sigue promoviendo la construcción de un bloque regional de poder político, con gobiernos revolucionarios de izquierda y convertidos en izquierda, anti-imperialistas y anti-neoliberales, dispuesto a desconstruir las bases sociales y económicas del capitalismo en sus diversas manifestaciones en sus respectivas sociedades. Es por ello que el presidente Chávez profundiza el ALBA con la creación de nuevos espacios públicos de integración y la legitimación de los que ya están creados, no subordinados a los lineamientos de las transnacionales y a la lógica del llamado Consenso de Washington, sino a los intereses de los pueblos, pretendiendo con la construcción del nuevo socialismo del siglo XXI, liberarlos de la dominación del capital y de las empresas explotadoras, sirviendo Venezuela de referencia para la generación de acciones gubernamentales orientadas a los reacomodos y cambios de los medios y modos de producción capitalistas al modelo de producción social, la colectivización de la economía y el de las empresas.

En el período 2004-2010, los triunfos democráticos de la nueva izquierda o centroizquierda en el continente, contribuyeron para que las estrategias de la diplomacia bolivariana, desplegadas por el gobierno venezolano de Chávez, obtuvieran resultados positivos en la conformación de los organismos especializados del ALBA, al despertar en ciertos gobiernos sudamericanos, centroamericanos y caribeños interés en el establecimiento de instancias multilaterales en las áreas energética, comunicacional y financiera, propuestas con una visión humanista, endógena y socialista, para superar las condiciones sociales y económicas, generadas por ola neoliberal que azotó al continente en las décadas de 1980 y 1990 (FERMIN, 2010), estas propuestas convertidas en realidad, son las siguientes: Petro-Caribe en junio de 2005, ${ }^{11}$

\footnotetext{
${ }^{11}$ Las comisiones especiales de Venezuela en las reuniones ministeriales con los representantes de los gobiernos caribeños exponen los beneficios de la propuesta energética. Es el 29 de junio de 2005 se celebra la I Cumbre de Petro-Caribe, en la cual se sella la creación del órgano multiestatal petrolero. Los países firmantes del acuerdo de cooperación energética fueron Belice, Jamaica, Cuba, San Cristóbal y Nieves, Antigua y Barbuda, Dominica, República Dominicana, Santa Lucía, San Vicente y Las Granadinas, Granada, Guyana, Bahamas, Surinam y Venezuela. Todos suscribieron el acuerdo energético, con las excepciones de Trinidad y Tobago y Barbados, "países productores de petróleo, que ante la evidente decepción de Chávez, expresaron sus reservas, ya que consideraron que podía afectar su propio acuerdo energético con los restantes países de CARICOM" (SERBIN, 2006: 87); luego se adherirían Haití (2007), Nicaragua (2007), Honduras (2008) y Guatemala (2008). En el primer considerando del acuerdo se establece como objetivo fundamental del órgano, "contribuir a la seguridad energética, al desarrollo socio-económico y a la integración de los países del Caribe, mediante el empleo soberano de los recursos energéticos, todo esto basado en los principios de integración denominada Alternativa Bolivariana para América (ALBA)" (PETRO-CARIBE, 2005).
} 
TeleSur en julio de 2005, ${ }^{12}$ Banco del ALBA en enero de $2008,{ }^{13}$ y, la creación de una zona monetaria común, que contará con una unidad de cuenta común, transacciones homologadas y fondos de compensación para adoptar el Sistema Único de Compensación Regional de Pagos (SUCRE) como moneda única, moneda virtual que podrá "circular" a partir de enero del 2010 (ALBA, 2009a), sin embargo, esto no significó en gran escala la ampliación de su membresía, sólo entre el 2007 y 2010 se incorporaron tres países miembros del primer organismo especializado mencionado -Nicaragua, Comunidad de Dominica y Honduras-, en Tele-Sur sólo Venezuela, Cuba, Bolivia y Nicaragua son socios accionarios de ésta empresa multiestatal, siendo la excepción el Banco del ALBA, todos sus miembros pertenecen al espacio unionista, $y$, en el caso del SUCRE, inició implementación sólo entre Venezuela, Ecuador y Bolivia.

De esto se infiere que las características de la diplomacia bolivariana, autonomía política, ideología propia, uso de las fuentes energéticas (gas y principalmente petróleo), las potencialidades comerciales y la atención primaria a los sectores más vulnerables de la población a través de la internacionalización de programas y las

\footnotetext{
${ }^{12}$ TeleSur, empresa multiestatal latinoamericana, entre al aire el 24 de julio de este mismo año, para enfrentar las empresas transnacionales mediáticas del capitalismo, llevar noticias e información emancipadoras de los pueblos; fundada por Venezuela, Argentina, Cuba y Uruguay, incrementaría su membresía a 8, con el ingreso accionario de Bolivia (2006), Nicaragua (2007), Ecuador (2007) y Paraguay el más reciente, el 15 de agosto de 2008. Para Cañizales y Lugo (2007, p.56) "Tele-Sur tuvo un doble objetivo. El primero era de servir de contrapeso a la dominación hegemónica de las cadenas internacionales como la BBC y CNN, mientras que el segundo era promover la integración política y cultural por medio del fortalecimiento de lo que ellos consideraban era la esfera pública común en la región. Esta dualidad refleja, en gran medida, los nuevos objetivos de la diplomacia venezolana, como el de apuntalar nuevas relaciones geográficas como forma de contrarrestar posibles políticas aislacionistas de Estados Unidos".

${ }^{13}$ Ante la crisis del sistema financiero internacional, por lo tanto, el deterioro de los organismos financieros multilaterales (FMI y BM), legitimadores del capitalismo financiero, para los miembros del ALBA era necesario una nueva alternativa monetaria y financiera. Los fundadores del espacio público unionista proponían desde el año 2004, que se hace realidad en la VI Cumbre del ALBA, el 23 de enero de 2008, cuando Venezuela, Cuba, Bolivia, Nicaragua y Comunidad de Dominica firman el Acta de Constitución del Banco del ALBA, para promover el desarrollo socio-productivo de los pueblos, eliminando el burocratismo propio del capitalismo financiero para procesar el financiamiento de los proyectos gran-nacionales. Honduras no se adherió a la entidad bancaria.
}

misiones sociales (Equipo de Investigación del Instituto de Altos Estudios Diplomático Pedro Gual, 2006), fueron definiendo el comportamiento de los gobiernos del continente a favor, en contra o neutrales al ALBA: los que decidieran su incorporación al espacio público unionista, parecía y sigue pareciendo más utilitarista que ideológica (anti-norteamericana).

Para el presidente Chávez el ALBA, como contrapropuesta al ALCA, no se limita sólo a las áreas geoestratégicas especificas que viene construyendo en sudamérica, centroamérica y caribeña; en las acciones políticas y diplomáticas opera la compactibilidad del proyecto unionista con otros espacios públicos de integración (GUERRA; MALDONADO, 2006), ya creados (MERCOSUR) y nuevos (UNASUR), con diferencias notorias en cuanto al enfoque que los miembros plenos implementaron a través del comercio y la inversión, compartiendo en cierta medida con ellos la forma como atienden con estos factores -comercio e inversión- y los ingresos que generan el desarrollo social de los pueblos; esos gobiernos promovieron, conjuntamente con los sectores empresariales correspondientes a las diferentes ramas de la economía, el intercambio comercial, principalmente en el caso de los miembros plenos del MERCOSUR, Argentina, Brasil, Uruguay y Paraguay; mientras que en la alianza bolivariana las relaciones de desarrollo social y humano entre los pueblos, subordina al mercado a la razón del Estado, más no lo elimina, ya que reconoce su valor en la globalización de la economía y la transnacionalziación de los bienes y servicios que circularon en las economías nacionales que definían la dinámica de este mercado. Además, el presidente Chávez propuso en la V Cumbre Extraordinaria del ALBA-TCP, celebrado en abril de 2009, crear mecanismos de cooperación multilateral con China e Irán (ALBA, 2009b), para promover las relaciones comerciales extra-continentales.

El presidente Chávez apuesta en el periodo 20042010 por la renovación de MERCOSUR y apoya la consolidación de la UNASUR. Según las afirmaciones del mismo Chávez, existen evidencias de que estos espacios públicos de integración rechazan la lógica de la economía de mercado, por ello, en reuniones privadas, paralela a las cumbres presidenciales de estos espacios, habilidosamente exhorta a algunos gobiernos a defender más los intereses del pueblo que del mismo mercado. En este sentido, para Chávez el ALBA representa una innovación social y humana en la consolidación del MERCOSUR y la 
UNASUR, donde el mercado queda a merced del Estado y el desarrollo económico está subordinado a la condición humana y a la autonomía del individuo de la lógica del capital global, que opera en la implementación de las políticas públicas generadoras de la igualdad de condiciones y oportunidades en áreas claves de la vida de los individuos: salud, educación, vivienda y seguridad social. El ALBA marca distancia con el PPP y silenciosamente, desde adentro, compite con el avance progresivo de la UNASUR, sin menoscabar el expansionismo y posicionamiento del espacio público unionista, por el contrario, reafirmaría su relevancia para la construcción del llamado "Nuevo Socialismo del Siglo XXI" y la lucha contra los Estados imperiales en un mundo convulsionado por la lógica del capital después del estallido de la crisis financiera mundial en septiembre de 2008 en los Estados Unidos. ${ }^{14}$

Esto explica como en el Enfoque Nueva Geopolítica Internacional del Primer Plan Socialista de la Nación 20072013, bajo los lineamientos del Proyecto Nacional Simón Bolívar 2007-2021, ${ }^{15}$ el presidente Chávez plantea que

La construcción de un mundo multipolar implica la creación de nuevos polos de poder que represente el quiebre de la hegemonía unipolar, en la búsqueda de la justicia social, la solidaridad y las garantías de paz, bajo la profundización fraterna entre los pueblos, su autodeterminación y el respeto a las libertades de pensamiento (MINISTERIO DE DESARROLLO Y PLANIFICACIÓN, 2007, p. 4).

Así, en las áreas de interés geoestratégicas, en función de orientar a la política exterior en la construcción de un nuevo bloque regional de poder político, definen en primer orden a América Latina y el Caribe. Para ello, el gobierno se propone la consecución de los siguientes objetivos (MINISTERIO DE DESARROLLO Y PLANIFICACIÓN, 2007, p. 42):

a) Participar en la construcción del nuevo MERCOSUR hacia la conformación de la Comunidad Sudamericana de Naciones, sobre la base de evaluación, revisión y reorientación de los contenidos de la integración.

${ }^{14}$ Ignacio Ramonet (2008). La crisis del siglo. El Fin de una era del Capitalismo Financiero. Caracas (Venezuela): Fundación Editorial el perro y la rana.

${ }^{15}$ Las Siete Líneas Estratégicas del Proyecto Nacional Simón Bolívar 2007-2013 es la primera etapa del Proyecto Nacional Simón Bolívar 2007-2021, anunciado en la campaña electoral correspondiente a los comicios presidenciales de diciembre de 2006. b) Consolidación del eje de liderazgo Cuba-Venezuela-Bolivia para impulsar el ALBA como alternativa al ALCA y a los TLC.

c) Fortalecer el esquema de integración suramericana, a través de la Comunidad Suramericana de Naciones, ampliando y consolidando las relaciones políticas, económicas y culturales en general con el Caribe. Entre los aportes del país para la cooperación y complementación, están las experiencias transitadas en el combate a la pobreza y la inclusión social.

d) Neutralizar la acción del imperio fortaleciendo la solidaridad y la opinión pública de los movimientos sociales organizados.

e) Fortalecer la alianza Venezuela, Suramérica y el Caribe.

El debilitamiento del liderazgo tradicional de Brasil y México en la región (González, 2006), a pesar de conservar su poder de influencia en ciertos espacios públicos de integración (SILVA, 2008), centrados más en sus propias propuestas de integración desde la óptica de la política doméstica, favoreció el posionamiento del ALBA, sin embargo, en el caso de Brasil buscaba fortalecer su liderazgo en la UNASUR, basándose en el desarrollo productivo, industrial, comercial y pragmatismo político en las relaciones bilaterales y multilaterales (FLÓREZ, 2009), para convertirse desde el continente en un referente regional y global como una potencia emergente en términos geopolíticos y económicos (SILVA, 2008), aún más con su participación en el G-20 (grandes exportadores de de productos agrícolas) y el IBSA (la iniciativa de cooperación entre India, Brasil y Suráfrica).

Los discursos y acciones de cooperación bolivariana del presidente Chávez edificaron al ALBA como un espacio público unionista, superando los desafíos políticos domésticos y aprovechando el aumento del petróleo en el mercado mundial, fue ampliando su membresía con el triunfo democrático de gobiernos de izquierda en América Latina, Bolivia, Nicaragua y Ecuador como se mencionó anteriormente. De las acciones gubernamentales de Chávez se fueron generando los primeros aportes políticos y diplomáticos para la construcción de un bloque regional de poder política, donde el concepto de política e ideología que opera en los promotores fundacionales, difiere totalmente de la concepción económica capitalista de la integración, predominante desde la década de 1950, concepto que cambia de dirección en el caso de los miembros de los organismos especializados. Esto significa que desde el ALBA los gobiernos miembros promueven el 
desarrollo sistémico de las sociedades, considerando los factores políticos, sociales, económicos, culturales, ecológicos e, incluso, ideológicos, como un sólo elemento transformador de las condiciones de pobreza y exclusión social que padece la población para que alcance un desarrollo económico humanista en el siglo XXI.

\section{El ALBA avanza al ritmo del surgimiento de la nueva izquierda}

Durante el período 1999-2003, el presidente Chávez presenta la propuesta unionista en los foros, universidades, parlamentos, empresarios y movimientos sociales latinoamericanos y caribeños, sin lograr resultados de ningún tipo. Se trata de que ningún gobierno expresó públicamente en las cumbres presidenciales su apoyo o respaldo a la creación de una alternativa de desarrollo social y humano diametralmente opuesta al ALCA, o, al "capitalismo salvaje", ni en las reuniones preparatorias de esas cumbres, en las cuales se discutió y aprobó el eje temático de la agenda de trabajo de los mandatarios y las declaraciones finales; la excepción sería Cuba, que ya tenía más de cuarenta años gritando en el desierto contra la dinámica de la integración económica, sin escuchar eco alguno. Por esto,

\begin{abstract}
Chávez fue articulando un nuevo mapa regional de alianzas y vínculos, entre los que se destacan la estrecha relación con Cuba (profundizada después del frustrado golpe de abril de 2002) y los nexos con los gobiernos progresistas y movimientos de izquierdas en América Latina y el Caribe. (SERBIN, 2006, p. 82)
\end{abstract}

En un escenario regional caracterizado por el surgimiento de la llamada nueva izquierda o centroizquierda, sobre todo entre el 2004 y 2010, que contribuyeron al fortalecimiento organizacional e institucional del ALBA, presentandose así el organismo con una "rostro claro y preciso" a la comunidad interamericana.

Es a partir del año 2006 que algunos gobiernos electos en el continente apoyaron el proyecto político revolucionario, al incorporarse al ALBA bajo la modalidad de relaciones bilaterales, y otros que celebraron acuerdos con Venezuela, fuera de los tradicionales espacios públicos de integración, no manifestaron públicamente interés en convertirse en miembros plenos de la alianza bolivariana, como se observa en la actitud de los gobiernos de Brasil, Argentina, Uruguay, Paraguay y de Ecuador hasta el año 2009, aliados estratégicos de la revolución bolivariana en el continente, o en el caso emblemático de los países miembros de Petro-Caribe, sólo cuatro de los 18 miembros del organismo, incluyendo a Venezuela, formaban parte del espacio público unionista, el resto se movían por el interés de la cooperación energética sin adjetivo político e ideológico. Para algunos analistas, académicos y políticos, esto demuestraba una renuncia de los gobiernos del Caribe a sumarse al ALBA, que se profundiza en la gran mayoría de ellos con las negociaciones de los tratados de libre comercio con los Estados Unidos $\mathrm{y}$ las posiciones en las cumbres internacionales, nada favorable a las propuestas venezolanas (SERBIN, 2006).

La instauración del ALBA en América Latina y el Caribe configura la formula bilateral con el ingreso de Bolivia (abril 2006), Nicaragua (enero 2007), Comunidad de Dominica (enero 2008), Honduras (agosto 2008) y Ecuador (junio 2009), previa para la construcción de un nuevo multilateralismo regional; sin duda necesitaba de un tratado constitutivo, aprobado por los parlamentos de los respectivos países miembros. En este sentido, se busca que el nuevo multilateralismo se vincule con la conquista de la izquierda del poder estatal de estos países, con un discurso anti-neoliberal y anti-norteamericano, demostrando que con la implementación de las directrices del Consenso de Washington en el continente aumentaron los índices de pobreza y la exclusión social, por lo que tienen que rechazar las pretensiones imperiales de los Estados Unidos, sobre todo Venezuela, Cuba, Bolivia, Nicaragua, Honduras y Ecuador, mientras que los países caribeños se mueven con cierto cuidado en las posiciones políticas-ideológicas. Estos gobiernos reconocieron el liderazgo continental de Chávez para la construcción del Gran Estado Americano a través de la unión de esfuerzos, potencialidades y debilidades.

Los primeros síntomas que revelaron la conformación de un bloque regional de poder en el hemisferio se comienza a observar con la llegada de gobiernos anti-imperialistas y anti-norteamericanos después del año 2005, y el ingreso de éstos al ALBA, legitimando el proyecto político bolivariano continental, quedando la variable económica condicionada a lo político-ideológico, ya sea de manera realista u oportuna de los gobiernos para aprovechar los beneficios financieros, energéticos y sociales de las políticas solidarias, complementarias y cooperativas que se implementaron desde el seno institucional del 
espacio público unionista. Las características políticas de estos gobiernos: afiliación ideológica, necesidad de construir un proyecto económico regional alternativo al modelo neoliberal y posición unitaria en temas claves del sistema interamericano e internacional.

Cabe destacar que el ingreso de Honduras al ALBA, previa solicitud el 30 de julio de 2008, en el Consejo Alimentario de Petro-Caribe, generó críticas por parte de los grupos políticos y sociales adversos al gobierno de Manuel Zalaya, al considerarlo un seguidor de los postulados de la revolución bolivariana, además, denunciaron la inhejerencia de Chávez en los asuntos propios de Honduras cuando el presidente Zelaya propone una "cuarta urna", en éste mecanismo el electorado decidiría por una Asamblea Nacional Constituyente al estilo de los cambios introducidos en Venezuela (1999), Bolivia (2009) y Ecuador (2009); convirtiendose en una de las prinicipales causas que activaron en los adversarios políticos de Zelaya el golpe de Estado en la madrugada del 28 de junio de $2009^{16}$, el mismo día de los comicios, con el apoyo de las fuerzas armadas y del tribunal supremo de justicia. En otras palabras, las relaciones estrechas de Zelaya con el presidente Chávez y la presencia de la revolución bolivariana en asuntos propios del gobierno, permitió a Honduras ingresar a Petro-Caribe y al ALBA, recibir beneficios económicos y sociales al país en la lucha contra la pobreza y las desigualdades sociales, convirtiéndose en centroamérica, conjuntamente con el gobierno nicaragüense de Daniel Ortega, en una pieza clave del avance la izquierda, atentando así contra los intereses económicos y políticos de la derecha, recurre

${ }^{16}$ Días antes, el 24 de junio de 2009, los miembros del ALBA, reunidos en la ciudad de Maracay (Venezuela), durante de la VI Cumbre Extraordinaria del ALBA-TCP, manifestaron su respaldo a la consulta popular, activada por el presidente Zelaya: "su firme compromiso de respaldar el proceso de consulta popular que por iniciativa del Presidente José Manuel Zelaya Rosales y del Gobierno del Poder Ciudadano, que está dirigida a responder a la genuina aspiración de la ciudadanía, que exige ser tomada en cuenta, mediante procesos de consulta y formas de expresión democráticas, que lejos de promover procesos dramáticamente violentos, aspira a construir una cultura de Paz y de No Violencia. De esa manera, la ciudadanía logrará un sistema de representación legítimo y recuperará el derecho a ser consultada permanentemente sobre los grandes temas o decisiones que en materia económica, política, social o cultural, afectan sus vidas y el devenir de la familia hondureña. Países miembros del ALBA - TCP enviarán acompañantes electorales que legitimen y den credibilidad a los resultados de este proceso de consulta popular ciudadana que se realizará el 28 de Junio de 2009" (ALBA, 2009d). a la salida no-democrática del presidente Zelaya del poder estatal. Los países miembros del ALBA rechazaron tal acción y exigieron el regreso inmediato de Zelaya a la Presidencia de Honduras, activaron los mecanismos de la OEA (ALBA, 2009c), sin resultados algunos.

Si bien los gobiernos de Brasil, Argentina y Uruguay pertenecen a la izquierda progresista, con fuertes relaciones económicas y energéticas con Venezuela, los del período 1999-2010, marcan distancia geo-estratégica con el ALBA, defienden su propio espacio público de integración, estructurado sobre la base de la globalización de la economía mundial, apuestan por el fortalecimiento y consolidación de la UNASUR, la fusión del MERCOSUR y la CAN en un solo bloque económico sub-regional con capacidad de competir con los demás bloques económicos mundiales; sin embargo, después de una larga espera, esto no afectó de que la solicitud del gobierno de Chávez de incorporar a Venezuela en el MERCOSUR, fue aceptada en diciembre de 2005 como miembro asociado, pero falta su aprobación en los parlamentos de Brasil y Paraguay, donde la oposición juega un papel clave en la balanza de peso y contra-peso en la relación parlamento-ejecutivo. No obstante, Chávez, con el enfoque de la ALBA, presenta propuestas orientadas al fortalecimiento institucional y geo-estratégico de la UNASUR, como la creación de Petro-Sur, la Universidad del Sur, el Banco del Sur y el Gaseoducto del Sur, y apoya vehementemente a la creación del Consejo de Seguridad del Sur, ya que concibe al nuevo espacio público de integración bajo el enfoque de las relaciones Sur-Sur y algunos de sus miembros plenos con la dirección de las relaciones Norte-Sur, los más evidente de esta apreciación son Chile, Colombia y Perú.

No se puede negar que la estrategia bolivariana se instaura al ritmo del surgimiento de la nueva izquierda o centroizquierda en los escenarios nacionales, por lo que su consolidación esta condicionada a la continuidad democrática en el tiempo de los formatos de gobiernos de izquierda, ya que la institucionalidad política e ideológica del ALBA no permitiría que se conserve con gobiernos de derecha en los países miembros, si estos llegaran a ganar los próximos comicios electorales; una de las primeras decisiones de esos gobiernos de derecha sería el retiro oficial de su país y en el caso de los organismos especializados, como Petro-Caribe y el Banco del ALBA, tendrían que cumplir con lo acordado en los acuerdos celebrados entre las partes, reconocer las deudas con 
Venezuela por el suministro de petróleo y la de la entidad bancaria por el financiamiento de proyectos grannacionales. Las tensiones políticas en Venezuela, Bolivia, Nicaragua, y, la situación Honduras, durante el golpe de Estado y después de las elecciones presdienciales del 29 de noviembre de 2009, la denuncia de magnicidio y golpes de Estado, ponen en peligro la consolidación del ALBA en el futuro inmediato, al punto de que el espacio público unionista pueda retroceder a diciembre de 2004, las relaciones estratégicas entre Venezuela y Cuba; el aumento de la membresía sería una de las formas de fortalecimiento institucional y la estabilidad del precio del petróleo en el mercado mundial para que Venezuela sigue promoviendo las relaciones económicas con justicia social y los mismos miembros de Petro-Caribe impulsando el desarrollo social con la forma de comercialización del petróleo venezolano, y el apoyo a otros países que sufren las consecuencias de las tragedias naturales, como sucedió en Haití en enero de 2010, activando así el ALBA el Plan de Ayuda para la Reconstrucción de Haití a través de la creación del "Fondo Humanitario del ALTA-TCP para Ayuda al Pueblo Haitiano" (ALBA, 2010a), y, criticaron la presencia militar en la isla (ALBA, 2010b).

En la IX Cumbre Presidencial del ALBA, celebrada en abril de 2010, los gobiernos de Antigua y Barbuda, Bolivia, Cuba, Dominica, Ecuador, Nicaragua, San Vicente y las Granadinas y Venezuela, declararon en el Manifiesto Bicentenario de Caracas, denominado "Consolidando la Nueva Independencia", que la alianza bolivariana

ha contrarrestado los efectos estructurales del capitalismo, construyendo una plataforma de unión e integración verdaderamente alternativa, que promueve la solidaridad, la cooperación, la complementariedad, el respeto, la justicia y la equidad, bases para la consolidación de espacios alternativos a las dinámicas y los mecanismos que hoy aseguran la hegemonía global del Capitalismo. (ALBA, 2010c)

Por esto los países miembros del ALBA alertaban:

[...] sobre el perverso papel desempeñado por importantes medios de difusión masiva al servicio de los intereses del imperialismo y en contra de los intereses y aspiraciones de los movimientos sociales y los pueblos del Tercer Mundo. Condenan el uso por parte de estos medios de la mentira, la distorsión, la calumnia y la omisión deliberada, amparados por el monopolio de los canales de comunicación y los grandes recursos financieros a su disposición. Rechazan la tendencia a la hipocresía y los dobles raseros de importantes medios informativos europeos y norteamericanos, cuyas respectivas políticas editoriales responden a objetivos enemigos de los Gobiernos revolucionarios y progresistas de América Latina y el Caribe y de los pueblos de la región (ALBA, 2010c).

Considerando el papel revolucionario de los medios de comunicación social de la era de la globalización de la economía, para el presidente Chávez el ALBA se convertía en el instrumento de lucha y liberación de los pueblos. Lograr la unidad de los Estados siginificaría el enfrentamiento simétrico con la hegemonía global del capitalismo y el poder imperial de los Estados Unidos, para así socanvar las bases sociales del Estado burgues representativo y seguir avanzando en el proceso de construcción del llamado Estado socialista, no sólo en los países miembros de la alianza bolivariana, sino también en aquellos que despierten de la pesadilla neoliberal.

\section{A Modo de Conclusiones: ¿Avances o retrocesos del proyecto político continental de Hugo Chávez?}

La búsqueda de un reacomodo y posicionamiento estratégico del gobierno de Chávez en los foros latinoamericanos y caribeños, fue revelando la situación de su proyecto político en el continente, primero, con las ideas confusa y difusas acerca de la Confederación de Estados y luego, en la precisión conceptual y operativa del ALBA, el desarrollo integral para los pueblos con un enfoque humanista, endógeno y socialista de las relaciones políticas, sociales, culturales y económicos de los pueblos, donde la liberalización del comercio de bienes y servicios, los flujos de capitales, la amortización de las políticas económicas y la compensación de las diferencias de productividad, serían procesadas bajos los parámetros de una economía justa y equitativa, hasta con el enfoque de economía de equivalencia (una forma de trueque). Este reacomodo y posicionamiento comenzó con la definición interna de la orientación de la revolución bolivariana, durante el período 2004-2010, experimentando el ALBA avances y retrocesos en las relaciones políticas y diplomáticas (petroleras y comerciales) con la gran mayoría de los países sudamericanos, centroamericanos y caribeños, que amerita una reorientación de las relaciones internacionales, con especial énfasis en la región, promoviendo en esas relaciones los fundamentos del llamado nuevo socialismo del siglo XXI. 
Si bien desde febrero de 1999 el gobierno revolucionario introduce cambios organizacionales en la estructura organizativa del Ministerio de Relaciones Exteriores, esto no significó en la práctica un cambio sustancial de la política exterior al ritmo de la intensidad con que el presidente Chávez delineaba su proyecto político continental en la escenario hemisférico, con especial énfasis en los foros latinoamericanos y caribeños en los que participaba y criticaba fuertemente los resultados de las políticas sociales del neoliberalismo. Por lo tanto, la diplomacia presidencial marcaba un paralelismo con la burocracia ministerial de la política exterior, que comenzaría a desaparecer después del retorno de Chávez al poder estatal, el 13 abril de 2002, aún más cuando supera las consecuencias políticas del paro petrolero de diciembre de 2002 a marzo de 2003, y, se evaporaría definitivamente con los resultados del referendo revocatorio del mandato presidencial, el 15 de agosto de 2004, al trazar los objetivos estratégicos y herramientas para lograrlos en la nueva etapa de la revolución; en el mapa estratégico fijado en la reunión de alto nivel, los días 11 y 12 de noviembre de este mismo año, aparece el ALBA como una acción política y diplomática de inmediata ejecución para que Venezuela inicie los pasos respectivos para la transformación del orden unipolar en la región, pasos que profundizaría después del 3 de diciembre de 2006 con la operaciolización de las acciones y estrategias delineadas en el Enfoque Nueva Geopolítica Mundial del Primer Plan Socialista de la Nación 2007-2013.

El ALBA amplía su membresía, de dos a ocho miembros plenos, con posibilidades de seguir aumentando, en la medida que triunfe la izquierda en los comicios electorales venideros y se decidan los gobiernos actuales a ingresar al espacio público unionista, siguiendo el ejemplo de los miembros de más reciente incorporación. En diciembre de 2004 solo Venezuela y Cuba eran sus miembros promotores; Bolivia se suma en abril de 2006, Nicaragua en enero de 2007, la Comunidad de Dominica en enero de 2008 y Honduras en agosto de 2008, con el triunfo de Lugo en Paraguay (2008), se pensó analiticamente que se convirtiría en uno de los nuevos integrantes del proyecto político continental, como lo hizo Ecuador, cuando el presidente Rafael Correa y su equipo de política exterior evaluó la solidaridad política y diplomática del gobierno de Chávez con su gestión, dejando a un lado el cuidado de la imagen del país andino ante los inversio- nistas extranjeros, como lo expresó el presidente Correa en el marco del inicio de la perforación del Pozo Bloque Ayacucho 5 de la Faja Petrolífera del Orinoco a finales de agosto de 2008, Ecuador esta observando cuidadosamente los avances y la consolidación del ALBA, por lo que "no descarta ingresar en este proyecto integracionista en un futuro cercano" (ALBA, 2008a), ingresando en junio de 2009, en respuesta de la solidaridad de Venezuela en la OEA ante la incursión colombiana en el territorio ecuatoriano, el 1 de marzo de 2008. Ante estas declaraciones, el gobierno venezolano destacó la importancia de éste país en el espacio público unionista, apostando por su futura adhesión (ALBA, 2008b).

Estos avances y retrocesos del ALBA dificultan que se convierta posiblemente en un nuevo modelo sistémico de unión política, social y económico, incluso militar, para América Latina y el Caribe, su mayor reto consiste en que los gobiernos de turnos la institucionalicen no sólo en sí misma, sino desde los diferentes niveles de poder del Estado, como se hizo en el pasado con los demás espacios públicos de integración fundados con la orientación de los postulados del modelo de desarrollo cepalino, modelo éste sustituido por el neoliberalismo. Probablemente uno de los miembros plenos genere la discusión de asumir la estrategia unionista de desarrollo en los niveles operativos de los espacios públicos de integración imperantes -MERCOSUR, CAN, SICA y CARICOM-, para llegar a una fusión de todos los espacios en uno sólo, el ALBA, o, que el UNASUR se convierta en un espacio público unionista con los postulados del ALBA, visión humanista, endógena y/o socialista, imponiéndose al enfoque de comercio e inversión que mueven en la UNASUR varios de sus miembros, como Brasil, Chile, Argentina, Colombia y Perú. O, las tensiones políticas domesticas en la relación oposición/gobierno afecte la continuidad del espacio público unionista, ya sea en Venezuela o en otro de los países miembros, más allá de los beneficios socio-económicos que reciben del país petrolero.

\section{Referencias}

AGUIRRE, Indalecio Liévano. Bolivarismo y monroísmo. Caracas/ Venezuela: Editorial Melvis, 2007.

ALIANZA BOLIVARIANA PARA LOS PUBLOS DE NUESTRA AMÉRICA (ALBA - TCP). Creado Fondo Humanitario del ALBA-TCP para Ayuda al Pueblo Haitiano. 2010a. Disponível em: < www. alternativabolivariana.com>. Acesso em: 10 dez. 2010. 
ALIANZA BOLIVARIANA PARA LOS PUBLOS DE NUESTRA AMÉRICA (ALBA - TCP). Declaración Reunión Extraordinaria del Consejo Político del ALBA. 2010b. Disponível em: < www.alternativabolivariana. com>. Acesso em: 10 dez. 2010.

ALIANZA BOLIVARIANA PARA LOS PUBLOS DE NUESTRA AMÉRICA (ALBA - TCP). Manifiesto bicentenario de Caracas, Declaración Final de la IX Cumbre del ALBA. 2010c. Disponível em: < www. alternativabolivariana.com>. Acesso em: 10 dez. 2010.

ALIANZA BOLIVARIANA PARA LOS PUBLOS DE NUESTRA AMÉRICA (ALBA - TCP). Intervención del Presidente Chávez en la $V$ Cumbre Extraordinaria del ALBA-TCP: Plenaria de la Cumbre Extraordinaria de Jefes de Estado y de Gobierno del ALBA-TCP. Cumaná/ Sucre: Museo de Arte Contemporáneo, 2009b. Disponível em: < www.alternativabolivariana.com>. Acesso em: 10 dez. 2010.

ALIANZA BOLIVARIANA PARA LOS PUBLOS DE NUESTRA AMÉRICA (ALBA - TCP). Declaración de los países miembros de la Alternativa Bolivariana para los Pueblos de Nuestra América en respaldo al proceso de consulta popular por iniciativa del Presidente Manuel Zelaya y del Gobierno del Poder Ciudadano. 2009c. Disponível em: < www.alternativabolivariana.com>. Acesso em: $10 \mathrm{dez} .2010$.

ALIANZA BOLIVARIANA PARA LOS PUBLOS DE NUESTRA AMÉRICA (ALBA - TCP). Declaración del Consejo Político sobre la situación en Honduras. 2009d. Disponível em: < www.alternativabolivariana.com>. Acesso em: 10 dez. 2010.

ALIANZA BOLIVARIANA PARA LOS PUBLOS DE NUESTRA AMÉRICA (ALBA - TCP). Declaración de la VII Cumbre ALBA-TCP. 2009e. Cochabamba/Bolivia: [S.n], 2009. Disponível em: < www.alternativabolivariana. com>. Acesso em: 10 dez. 2010.

ALIANZA BOLIVARIANA PARA LOS PUBLOS DE NUESTRA AMÉRICA (ALBA - TCP). Presidente Correa no descarta ingreso de Ecuador al ALBA. 2008a. Disponível em: < www.alternativabolivariana.com>. Acesso em: 27 ago. 2008.

ALIANZA BOLIVARIANA PARA LOS PUBLOS DE NUESTRA AMÉRICA (ALBA - TCP). Ecuador es necesario para el fortalecimiento del ALBA. 2008b. Disponível em: < www.alternativabolivariana.com>. Acesso em: 27 ago. 2008.

BICHARA, Julimar da Silva. Política exterior brasileña: multilateralismo e integración. Política Exterior, Madrid/ España, v. 22, n. 126, p. 93-103, nov./dez. 2008.
CAÑIZALES, Andrés; LUGO, Jairo. TeleSur: estrategia geopolítica con fines de integración. Confines, Monterrey/ México, v.3, n.6, p. 53-64, ago./dez. 2007.

COMISIÓN ECONÓMICA PARA AMÉRICA LATINA Y EL CARIBE (CEPAL). Anuario estadístico de América Latina y el Caribe 2000. Disponível em: <www.cepal. com>. Acesso em: 29 maio 2008.

CONSELHOMINISTERIALDECOMPLEMENTACIÓN ECONÓMICA DEL ALBA-TCP, 1., 2009, Caracas. Anais... 2009a. Disponível em: < www.alternativabolivariana. com>. Acesso em: 10 dez. 2010.

EL TROUDI, Halman. El Salto Adelante. La nueva etapa de la Revolución Bolivariana. Caracas/Venezuela: Ediciones de la Presidencia de la República, 2005.

EQUIPO DE INVESTIGACIÓN DEL INSTITUTO DE ALTOS ESTUDIOS DIPLOMÁTICOS PEDRO GUAL. La Cooperación Internacional Bolivariana en el año 2006: avanzando hacia la Pruripolaridad. Revista Política Exterior y Soberanía, Caracas/Venezuela, n. 3, ano 1, p. 25-32.

ESCORCIA, Reinaldo et al. Chávez universal, Caracas/ Venezuela: Revista Patria Grande, 2006.

FERMIN, F. Eudis F. (2009). La Alternativa Bolivariana para los Pueblos de Nuestra América: ¿La ruptura paradigmática de los modelos de integración económica? Revista Espacio Abierto, Macaibo/Venezuela, v.18, n.2, p. 339-365, abr./jun. 2009.

FERMIN, F. Eudis F. La Crisis del Ciclo Neoliberal en los Países de la Región Andina. Revista de Ciencias Sociales, Maracaibo/Venezuela, v.16, n. 4, p. 687-698, out./dez 2010.

Flórez, Guillermo Pérez. Unasur: la apuesta de Brasil. Política Exterior, Madrid/Espanã, v.23, n. 127, p. 149-160, jan./fev. 2009.

GONZÁLEZ U., Edmundo. Las dos etapas de la política exterior de Chávez. Nueva Sociedad, Caracas/Venezuela, n. 205, p. 159-171, set./out. 2006.

GUERRA, Sergio; MALDONADO, Alejo. Laberintos de la integración latinoamericana. Caracas/Venezuela: Pixels Computación, 2006.

MARTÍNEZ, Ricardo. El panamericanismo: doctrina y práctica imperialista: las relaciones internacionales desde Bolívar hasta Eisenhower. Buenos Aires: Editorial Alumine, 1957. 
MINISTERIO DE DESARROLLO Y PLANIFICACIÓN (2008). Líneas Generales del Plan de Desarrollo Económico y Social de la Nación 2007-2013. Caracas/ Venezuela, 2008. 1 CD-Rom.

MINISTERIO DE DESARROLLO Y PLANIFICACIÓN. Líneas Generales del Plan de Desarrollo Económico y Social de la Nación 2001-2007. Caracas Venezuela, 2001. 1 CD-Rom.

MOVIMIENTO QUINTA REPÚBLICA 1998. La Propuesta de Hugo Chávez para Transformar a Venezuela. Caracas/Venezuela: MVR, 1998.

MOVIMIENTO QUINTA REPÚBLICA 2000. La Propuesta de Hugo Chávez para continuar la revolución. Caracas/Venezuela: MVR, 2000.

NAÍM, Nayllivis; FERMÍN, Eudis. La política exterior del nuevo gobierno venezolano: continuidad y discontinuidad en el tema integracionista. Anuario de Cultura Latinoamericana, Pagani/Itália, n.4, p. 273-303, 2002.

OSSA, Álvaro de la. Cooperación e integración entre gobiernos en Centroamérica. Nueva Sociedad, Caracas/ Venezuela, n. 186, p. 60-68, jul./ago. 2003.

PARAMIO, Ludolfo. Giro a la izquierda y regreso del populismo. Nueva Sociedad, Caracas/Venezuela, n.205, p. 62-74, set./out 2006.

PETRAS, James. América Latina: movimientos, cambios y gobiernos de centroizquierda. Caracas/Venezuela: Monte Ávila Editores Latinoamericana, 2008.
PETRO-CARIBE. Acuerdo de Cooperación Energética Petro-Caribe. 2005 Disponível em: <www. alternativabolivariana.com $>$. Acesso em: 27 jan. Acesso em: 27 jan. 2008.

RAMONET, Ignacio. La crisis del siglo: el fin de una era del Capitalismo Financiero. Caracas/Venezuela: Fundación Editorial el perro y la rana, 2008.

ROMERO, Carlos. Jugando con el globo: la política exterior de Hugo Chávez. Bogotá/Colombia: Ediciones B., 2006

RUIZ, José Briceño. El ALCA, el ALBA y la política de integración de Venezuela. Revista del Banco Central de Venezuela, Caracas, v. 21, n. 2, p. 21-45, 2007.

SANDOVAL, Juan Manuel. El Plan Puebla-Panamá y el Plan Colombia. In: SANDOVAL, Juan Manuel; ÁLVAREZ, Raquel (Comp.). Integración y fronteras en América Latina. Mérida/Venezuela: ULA, 2003.

SERBIN, Andrés. Cuando la limosna es grande: el Caribe, Chávez y los límites de la diplomacia petrolera. Nueva Sociedad, Caracas/Venezuela, n. 205, p. 75-91, sep./oct. 2006.

VITIER, Cintio. Vida y obra del Apóstol José Martí. La Habana/Cuba: Fondo Cultural del ALBA, 2006. 